\title{
Aircraft 6-DOF Modular Modeling Based on MATLAB Simulink
}

\author{
Huixian Wang ${ }^{a}$, Dongli Mab \\ School of Beihang University, Beijing 100000, China \\ awanghuixian001@outlook.com, bklinge01@126.com
}

Keywords: aircraft, 6-DOF modeling, Matlab Simulink, modular principle.

\begin{abstract}
Aircraft is playing an increasing important role in aerospace field. To analysis the property of an aircraft, a model and simulation which is accurate and succinate is necessary. In this paper, 6DOF aircraft full motion equations are derived on the basis of newton's second law in the condition of plane earth hypothesis and rigid body hypothesis. Matlab Simulink was adopted to modeling simulation in which modular principle was employed. And the simulation results were reliable though the qualitative analysis.
\end{abstract}

\section{Introduction}

Aircraft is playing an increasing important role in aerospace field in which functions cover war, sightseeing, transportation and many other areas. Flight performance analysis plays an important role in the design of aircraft. And model building is a fundamental process. Because of the high cost of building and flight testing a real aircraft, the importance of aircraft mathematical models goes far [1]. Because the model is tightly integrated with pneumatic data, the simulation of aircraft in design phase is usually linearly simplified. But the development of modeling software makes the whole equation simulation possible. The modular modeling principle can make the model easy to modify and closely joint with the external software.

\section{6-DOF Modeling}

The differential equations are 12th order when the aircraft are seemed as rigid body, and it can be simplified to 2 or even 1 order according to target problem. The law of flight mechanics is determined by the special flight environment. In this paper, the equations of motion, which strictly derived by the Newton's second law, are composed of 3th order centroid rotation kinetic equations, 3th order centroid motion kinetic equations, 3th order geometric relations equations and 3th order inertial navigation equations [2]. Modeling is based on three assumptions as follows:

1) The earth is seemed as flat, and curvature and autobiography were ignored.

2) The aircraft is treated as a rigid body, regard the elastic deformation of the body and the influence of the rotating parts.

3) Ignoring machining errors, the aircraft is perfectly symmetrical about the longitudinal symmetry plane.

According to Newton's second law: $F=m a$.The external force is composed of thrust, gravity and aerodynamic force which decomposed as lift, resistance force and side force. Expand the centroid motion kinetic equations into a scalar system, as follows:

$$
m\left\{\left[\begin{array}{c}
\dot{u} \\
\dot{v} \\
\dot{w}
\end{array}\right]+\left[\begin{array}{ccc}
0 & -r & q \\
r & 0 & -p \\
-q & p & 0
\end{array}\right] \times\left(\begin{array}{c}
u \\
v \\
w
\end{array}\right)\right\}=\left[\begin{array}{c}
T \\
0 \\
0
\end{array}\right]+\left[\begin{array}{ccc}
\cos \alpha \cos \beta & -\cos \alpha \sin \beta & -\sin \alpha \\
\sin \beta & \cos \beta & 0 \\
\sin \alpha \cos \beta & -\sin \alpha \sin \beta & \cos \alpha
\end{array}\right]\left[\begin{array}{c}
-D \\
C \\
-L
\end{array}\right]+\left[\begin{array}{c}
-m g \sin \theta \\
m g \cos \theta \sin \phi \\
m g \cos \theta \cos \phi
\end{array}\right]
$$

According to the theorem of moment of momentum: $\mathrm{M}=\mathrm{dh} / \mathrm{dt}$. And according to the assumption, aircraft is symmetrical about the longitudinal plane, $\mathrm{I}_{\mathrm{XY}}=\mathrm{I}_{\mathrm{YZ}}=0$. Expand the centroid rotation kinetic equations into a scalar system, as follows: 


$$
\left[\begin{array}{ccc}
I_{x} & 0 & -I_{x z} \\
0 & I_{y} & 0 \\
-I_{x z} & 0 & I_{z}
\end{array}\right] \cdot\left[\begin{array}{c}
\dot{p} \\
\dot{q} \\
\dot{r}
\end{array}\right]+\left[\begin{array}{ccc}
0 & -r & q \\
r & 0 & -p \\
-q & p & 0
\end{array}\right] \cdot\left[\begin{array}{ccc}
I_{x} & 0 & -I_{x z} \\
0 & I_{y} & 0 \\
-I_{x z} & 0 & I_{z}
\end{array}\right] \cdot\left[\begin{array}{c}
p \\
q \\
r
\end{array}\right]=\left[\begin{array}{c}
L \\
M \\
N
\end{array}\right]
$$

The Euler angles constraint equations are given as follows:

$$
\left[\begin{array}{c}
p \\
q \\
r
\end{array}\right]=\left[\begin{array}{c}
\dot{\phi} \\
0 \\
0
\end{array}\right]+L_{x}(\phi)\left[\begin{array}{c}
o \\
\dot{\theta} \\
0
\end{array}\right]+L_{x}(\phi) L_{y}(\theta)\left[\begin{array}{c}
0 \\
0 \\
\dot{\psi}
\end{array}\right]
$$

The inertial navigation equations are the kinematic equations of centroid. From the kinematics law we get: $d x / d t=v$.Expand the kinematics law in body axial system:

$$
\left[\begin{array}{c}
\dot{x} \\
\dot{y} \\
\dot{z}
\end{array}\right]=\left[\begin{array}{ccc}
\cos \theta \cos \psi & \sin \theta \sin \phi \cos \psi-\cos \phi \sin \psi & \sin \theta \cos \phi \cos \psi+\sin \phi \sin \psi \\
\cos \theta \sin \psi & \sin \theta \sin \phi \sin \psi+\cos \phi \cos \psi & \sin \theta \cos \phi \sin \psi-\sin \phi \cos \psi \\
-\sin \theta & \sin \phi \cos \theta & \cos \phi \cos \theta
\end{array}\right] \cdot\left[\begin{array}{c}
u \\
v \\
w
\end{array}\right]
$$

The movement of the aircraft can be described through the summarization of the above equations. In which: ( $\mathrm{u} \mathrm{v} \mathrm{w)}$ are the projection of airspeed in body axial system; ( $\mathrm{p} \mathrm{q} \mathrm{r)}$ ) are the projection of angular velocity in body axial system; ( $\mathrm{L}$ D C)are the aerodynamic component defined in the airflow coordinate system; $(\alpha \beta)$ are attack angle and side slip angle; $\mathrm{mg}$ is gravity.( $\mathrm{L} \mathrm{M} \mathrm{N})$ are the projection of torque in body axial system; $\left(I_{X} I_{Y} I_{Z}\right)$ are moment of inertia; $I_{X Z}$ is product of inertia. $(\varphi \theta \psi)$ describe the attitude of the aircraft.

\section{Model Establishment in Simulink}

Matlab Simulink is adopted to build simulation model, and modularization is implied by subsystems block. The model consists of a force calculation module, an equation module which Programming with s-function, four input quantities and 10 output quantities [3]. The four input are elevator, aileron, rudder and engine speed. The ten output are $\left[\begin{array}{lllllllllllllll}u & v & w & p & q & r & \phi & \theta & \psi & h & V & \alpha & \beta\end{array}\right]$. The simulation model is shown below:

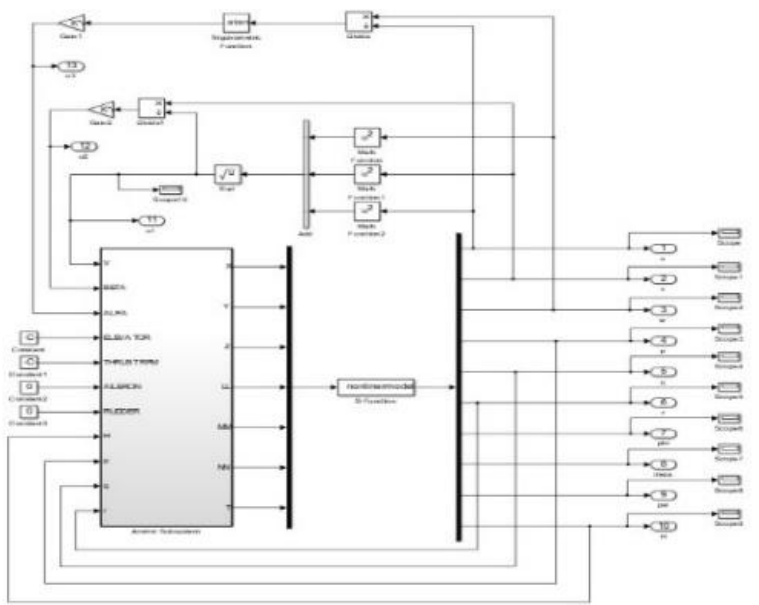

Fig. 1 simulation model

Force calculation part enjoy four input same as the whole model, and seven input quantities through feedback [V alpha beta h p q r], and it output seven quantities [L D C LL M N T]. The inner subsystem is shown below: 


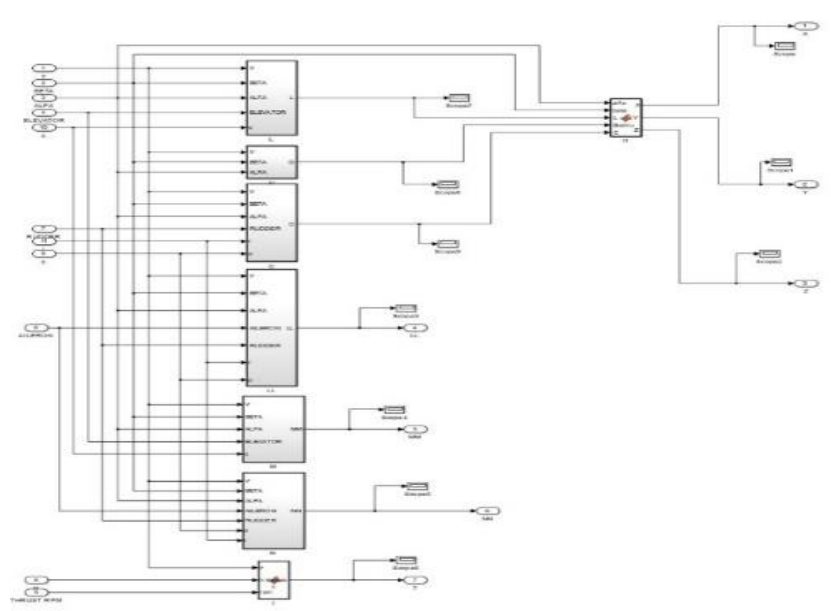

Fig. 2 inner subsystem

The aerodynamic data are saved in corresponding part respectively. For example, we can get engine thrust by enter height, velocity and thrust speed into T function as shown in figure. Blocking pneumatic data helps to simplify the modeling process with the associated software, such as fluent. Take the engine thrust as an example, the engine thrust in different height, engine speed and velocity, are given in tabular form, then we can import the table into the function and get the thrust by interpolation.

Although there is build-in aerospace block set, the equation block cannot easily modify. So we choose s-function to build the differential equation model. S-function is a function template for matlab. Declare the input and output, set the initial value and type the differential equation which derived in the previews chapter in corresponding flag in the function, then we get the model.

\section{Simulation Analysis}

The modeling of this paper is based on a high speed drone. Best cruise speed is $350 \mathrm{~km} / \mathrm{h}$, and the cruise height is $1500 \mathrm{~m}$.

The system should be trimmed first before the response is calculated. Take the reference state as the aircraft in best cruise state without slip. At this time the angle of attack is equal to the pitch angle. Only three degrees of freedom need to be considered. And the corresponding equation is:

$T \cos ($ alfa $)=D ; \quad L+T \sin ($ alfa $)=G ; \quad M=0$

Through the interpolation, first we find the elevator angle with zero pitch moment factor and the elevator angle with required lift factor in every attack angle, then seek the attack angle and elevator angle which Meet the above two condition simultaneously. trim results in the choose reference cruise state are:

$$
\text { alfa }=3.2011^{\circ} ; \text { elevator }=0.29939^{\circ} ; \quad \text { rpm }=74148
$$

The longitudinal response caused by the elevator deflection is taken as an example. Plus 1 degree on the elevator angle on the trimmed model, then set the time span as 200 second, and press the start key [4]. The results are as follows:
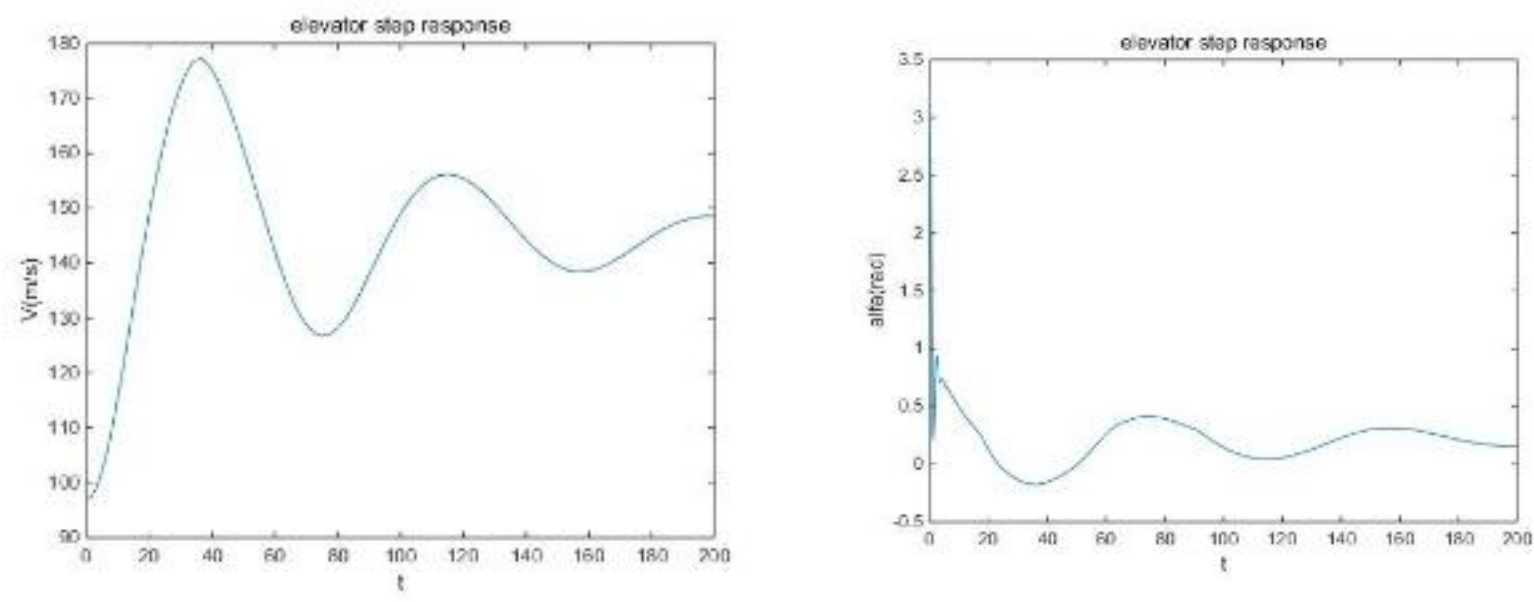

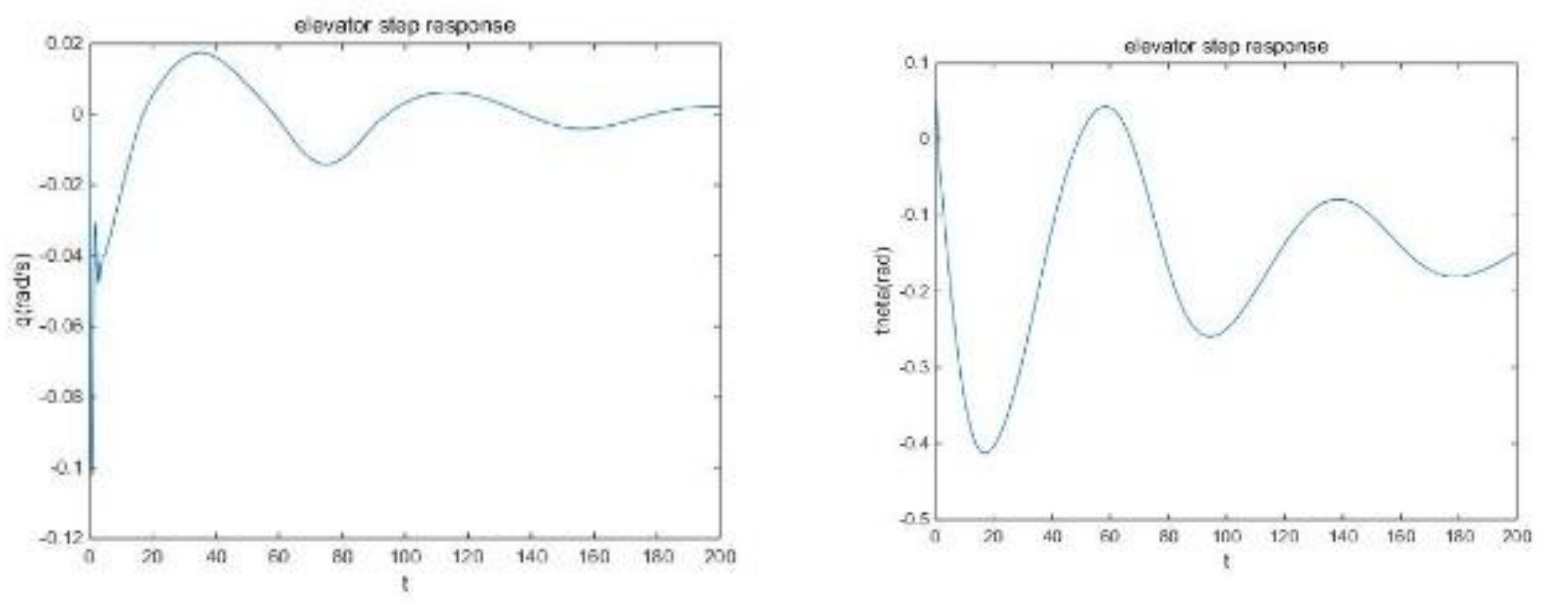

Fig.3 simulation result

Above four figures are the real-time simulation of velocity, attack angle, pitch angle speed and elevation speed. Through the non-lineation model, aircraft simulation can be more reliable and succinate.

\section{Summary}

In this paper, 6DOF aircraft full motion equations are derived on the basis of newton's second law in the condition of plane earth hypothesis and rigid body hypothesis. Matlab Simulink was adopted to modeling simulation in which modular principle was employed. The modeling method used in this paper has the following advantages:

1) The model equation is deduced according to the target situation. The factors that can be ignored are simplified.

2) Making the model more concise and clear through applying Matlab Simulink for modeling,

3) Making the connection between model and external software more simple and convenient through the implementation of the modular principle.

\section{References}

[1] Jun Xiao,Weiwei Zhang, Yujie Han, Min Sun. Influencing Factor and Corresponding Method of Target Drone Dynamic Stability

[2] Brian L. Stevens, Frank L. Lewis, Aircraft Control and Simulation, 2004

[3] Ong C M. Dynamic Simulation of Electric Machinery Using MATLAB/Simulink [J]. Using Matlab/Simulink, 1998.

[4] Hebert J, Keith J, Ryan S, et al. DGPS Kinematic Carrier Phase Signal Simulation Analysis for Precise Aircraft Velocity Determination [J]. Proceedings of Annual Meeting of the Institute of Navigation, 1997, 44(2): 231-245. 